\title{
Application of Convex Programming to Rescheduling of Transactions in a Deregulated Power Market
}

\author{
A.M. STANKOVIC ${ }^{\dagger}$ \\ B.C. Lesieutre ${ }^{\dagger \dagger}$ \\ A. MegretsKi ${ }^{\dagger \dagger}$
}

\begin{abstract}
In this paper we address the issue of improving transmission security in a deregulated power market. We propose an optimization procedure that assures that transmission security is maintained, and generates minimal corrections in existing contractual transactions that are necessary to ensure security. Our procedure is based on a DC load flow, and as such it is intended as a screening tool, and a source of candidate control actions that will be later refined by a more accurate (AC) model. Our approach may also enable faster calculations of important operational quantities like Available Transfer Capability (ATC).
\end{abstract}

Key. Words -convex programming, power systems, linear matrix inequalities (LMI).

\section{INTRODUCTION}

The deregulation of power systems around the world represents a fundamental change in the operation of a mature industry. It is arguably motivated to improve efficiency and lower consumer rates by promoting competition among generating entities, and it is supported by technological advances in combined cycle generation, flexible ac transmission, and more. A significant challenge is to introduce mechanisms for widespread trade in electricity that take into account characteristics of the physical system. (The interested reader might see [1] for discussions on a range of control and market issues associated with changes in the industry.)

Of particular concern is the ability of portions of the transmission grid to allow transmission of power from (possibly distant) generating stations through to (possibly distant) loads. To facilitate and promote commercial use of the system, "available transfer capability (ATC)" has been introduced as an economic measure of the of the power that may be transmitted through particular subsystems to sell to distant consumers $[2,3]$. Each transmission company (in the US) is required to supply the results of ATC calculations on the worldwide-web. ATC is intended to be used as an economic tool and does not account the effect of all possible disturbances, especially those occuring outside the domain of the transmission company performing the calculation. To ensure reliability, a overseeing system operator must evaluate the proposed market-based power contracts for feasibility on the physical system. This evaluation must determine whether or not the proposed power flows will violate any system constraints, and importantly, whether or not the system will remain secure after any number of likely disturbances.

There are, of course, many approaches to alleviating an overly-stressed power grid in operation. One marketbased approach is to simply curtail transactions when a part of the system is overloaded. (One method for analysis of such an approach is reported in [4].) In some scenerios, participants might bid to have their transaction honored. Another approach is to allow the operator to adjust transactions, to maintain safe operation. In this paper we follow the latter approach and employ convex programming techniques to determine minimal changes to transactions to ensure security.

In this development we assume that market participants, denoted here as generators and loads, have already decided on energy transactions for the period of interest. A role of the system operator is then to validate the technical feasibility of the network operation as agreed by the market participants. If adjustments are necessary, the system operator is to make sure that the deviations are as small as possible, and that effects of uncertainties in operation are shared among market players in an equitable and fair manner. We thus propose a method that is minimally invasive in the sense that it minimally modifies the nominal operating point proposed by participants to ensure system security (against a set of known credible disturbances). To achieve this goal, the analysis methodology used by the operator has to deal not only with optimization, but also with sensitivity of the proposed solution to typical system uncertainties.

\section{Problem Formulation}

Our problem formulation is based on the linearized (DC) power flow model for the network. The so generated solutions may be used in as a screening tool, and serve as a source of candidate control actions that will be later refined by a more accurate $(\mathrm{AC})$ model.

Consider the system with $G$ generating entities (denoted as generators for simplicity) and $D$ distribution entities; assume that the interconnection contains the total of $\breve{L}$ lines, all loads and generators are connected at all times, and that line flow constraints $\bar{y}$ are symmetric in both directions. It is assumed that each mem- 
ber of a subset of $L$ lines can be inoperable at a given time, so the security will be ascertained by evaluating $K$ configurations, each with $L=\breve{L}-1$ (or fewer) elements. We assume that the $D$-dimensional vector of loads $p_{d}$ is known, and not subject to rescheduling; the task is thus to check if the $G$-dimensional vector of generation $\breve{p}_{g}$ results in feasible and secure operation. If not, it is to be minimally altered (according to an adopted criterion) by adding a correction vector $\breve{x}$, so that $\breve{p}_{g}+\breve{x}$ results in both feasible and secure operation. It is assumed that each candidate vector $\breve{p}_{g}$ satisfies the energy balance, in that sum of its entries equals the sum of entries of the load vector, i.e.

$$
\mathbf{1}^{T} \breve{p}_{g}=1^{T} p_{d}
$$

where 1 is are vectors of ones of appropriate dimensions; then $1^{T} \breve{x}=0$, so only $G-1$ components of $\breve{x}$ are to be determined; we denote the first $G-1$ components of $\breve{x}$ as $x$, and first $G-1$ components of $\breve{p}_{g}$ as $p_{g}$.

We consider three types of operating constraints: 1) generation constraints, 2) line flow constraints and 3) overall active power loss constraint.

Generation constraints can be written as a $G$-vector:

$$
\breve{p}_{\text {min }} \leq \breve{p}_{g}+\breve{x} \leq \breve{p}_{\text {max }},
$$

or in terms of linear constraints on $\breve{x}$

$$
\breve{p}_{m}=\breve{p}_{\text {min }}-\breve{p}_{g} \leq \breve{x} \leq \breve{p}_{M}=\breve{p}_{\text {max }}-\breve{p}_{g},
$$

which is easily written in terms of linear constraints on $x$ :

$$
p_{m} \leq x \leq p_{M}
$$

Line flow constraints stem from graph-theoretic properties of the transmission network which establish that (given $L$ lines in operation, and standard nondegeneracy assumptions) there are $L-(G+D-1)$ loops in the network, so line flows $y$ can be written as:

$$
\underbrace{\left[\begin{array}{c}
A_{\text {loop }} \\
A_{\text {load }} \\
A_{\text {gen }}
\end{array}\right]}_{A} y=\left[\begin{array}{c}
0 \\
p_{d} \\
p_{g}
\end{array}\right]+\left[\begin{array}{l}
0 \\
0 \\
x
\end{array}\right] .
$$

In this equation, $A_{\text {loop }}$ is a $L-(D+G-1) \times L$ matrix corresponding to the loop flows; entries of $A_{\text {loop }}$ proportional to transmission line impedances (which in turn are very well approximated by reactances in extra high-voltage networks). Matrices $A_{\text {load }}(D \times L)$ and $A_{\text {gen }}((G-1) \times L)$ are incidence-type arrays, i.e., their entries are zeros and \pm 1 corresponding to nodal flow balances. Given typical availability of network information in power systems, matrix $A_{\text {loop }}$ is uncertain, as line parameters often drift during operation. Given (5),

$$
-\bar{y} \leq \underbrace{A^{-1}\left[\begin{array}{c}
0 \\
p_{d} \\
p_{g}
\end{array}\right]}_{\hat{y}}+\underbrace{A^{-1}\left[\begin{array}{l}
0 \\
0 \\
x
\end{array}\right]}_{\bar{y}} \leq \bar{y} .
$$

The entries of $A^{-1}$ are the injection coefficients that describe the sensitivities in line flows to variations in injections If we denote the last $G-1$ columns of $A^{-1}$ with $C$, the constraint (6) can be rewritten in a form that is linear in $x$ :

$$
y_{m} \leq C x \leq y_{M}
$$

where $y_{m}=-\bar{y}-\hat{y}$ and $y_{M}=\bar{y}-\hat{y}$.

Loss constraint may be placed in some cases on total system real power losses. This would correspond, for example, to the case when an independent system operator (ISO) has to meet the efficiency constraint; in terms of our notation

$$
(\hat{y}+C x)^{T} R(\hat{y}+C x) \leq W .
$$

The matrix of line resistances $R$ is diagonal and positive definite; inverses of its entries are often proportional to susceptance terms that are used in $A_{\text {loop }}$.

Several metrics $I(\breve{x})$ could be used to measure $\breve{x}$; among the commonly ones are $\|\breve{x}\|_{2}^{2}=\breve{x}^{T} \breve{x}$, and $\|\breve{x}\|_{1}=$ $\sum_{k}\left|\breve{x}_{k}\right|$. The $\|\cdot\|_{2}^{2}$ norm is often directly related to power or energy in engineering systems, and is a reasonable measure in many cases. Its form is also analytically convenient in optimization problems. The $\|\cdot\|_{1}$ norm is applicable to problems in which the absolute difference between quantities is considered critical. It is arguable in our formulation of this problem that differences in scheduled power correspond directly to revenue; the $\|\cdot\|_{1}$ norm may be appropriate in this case. In practice, it is possible to add weights to criterion functions to properly reflect economic and other terms that are important to a system operator.

Finally, the optimization problem we consider is:

$$
\min I(\breve{x})
$$

subject to the following constraints:

$$
\begin{aligned}
\breve{p}_{m} & \leq \breve{x} \leq \breve{p}_{M} \\
y_{m} & \leq C x \leq y_{M} \\
(\hat{y}+C x)^{T} R(\hat{y}+C x) & \leq W
\end{aligned}
$$

A set of constraints, (11) and (12), is appended for each of the $K$ network configurations that are included in the security analysis.

\section{Solution Method}

For solution of this programming problem with linear or quadratic criterion, and with linear and quadratic constraints we use the set of convex optimization procedures denoted in the control literature as LMI (linear matrix inequalities). Efficient interior point algorithms for solving such problems were developed in early nineties, and applied with great success to a number of control design problems. Its important advantage 
is that addition of constraints in (10)-(12) to account for various network configurations in security studies results in a not more than polynomial growth of computation times, and may actually improve the performance of the optimization procedure [5].

The main mathematical objects in LMI are matrices of definite signs, so various criteria (9) and quadratic constraint (12) have to be transformed to more convenient forms. In the case of $I(\breve{x})=\|\breve{x}\|_{2}^{2}=\breve{x}^{T} \breve{x}$, the criterion is replaced with a scalar minimization $\min \lambda$, with the additional constraint

$$
\left[\begin{array}{cc}
\lambda & \breve{x}^{T} \\
\breve{x} & I_{\breve{x}}
\end{array}\right]>0
$$

where $I_{\breve{x}}$ is a $G$-dimensional identity matrix.

In the case of $I(\breve{x})=\|\breve{x}\|_{1}=\sum_{k}\left|\breve{x}_{k}\right|$, the criterion is replaced with $\min \sum_{k} \lambda_{k}$, with

$$
\begin{aligned}
\breve{x}_{k} & \leq \lambda_{k} \\
-\breve{x}_{k} & \leq \lambda_{k} .
\end{aligned}
$$

The constraint on losses (12) is replaced with

$$
\left[\begin{array}{cc}
W R^{-1} & \hat{y}+C x \\
(\hat{y}+C x)^{T} & 1
\end{array}\right]>0
$$

\section{AN INTRODUCTORY EXAMPLE}

In Fig. 1 we show a four bus example; circled numbers are bus numbers; numbers in rectangles next to lines denote line flow constraints (i.e., entries of $\bar{y}$ ); arrows indicate generation (into a bus) and loads (away from a bus); branch reactances are denoted with a $j$ next to the value. In this example $G=2$ (buses 1 and 2 ), $D=2$ (buses 3 and 4 ), $L=5$. For security analysis, we consider the nominal configuration, and $K=4$ additional configurations (single outages of lines $1-2,1-4,2-3$ and 3-4). At the nominal operating point $p_{d}=\left[\begin{array}{ll}1 & 2\end{array}\right]^{T}, \breve{p}_{g}=\left[\begin{array}{ll}1 & 2\end{array}\right]^{T}, x$ is a scalar, $\breve{p}_{\min }=\left[\begin{array}{ll}0 & 0\end{array}\right]^{T}$ and $\breve{p}_{\max }=\left[\begin{array}{ll}3 & 3\end{array}\right]^{T}$. There are two loops in this example, so that (with lines ordered by increasing bus numbers, i.e., $1-2,1-4,2-3,2-4,3-4)$ the $A$ matrix is composed of:

$$
\begin{aligned}
A_{\text {loop }} & =\left[\begin{array}{ccccc}
1 & -2 & 0 & 1 & 0 \\
0 & 0 & 1 & -1 & 1
\end{array}\right] \\
A_{\text {load }} & =\left[\begin{array}{ccccc}
0 & 0 & 1 & 0 & -1 \\
0 & 1 & 0 & 1 & 1
\end{array}\right] \\
A_{\text {gen }} & =\left[\begin{array}{lllll}
1 & 1 & 0 & 0 & 0
\end{array}\right]
\end{aligned}
$$

Also from Fig. 1, line flow constraints are $\bar{y}=$ $\left[\begin{array}{lllll}2 & 0.9 & 2 & 2.5 & 1\end{array}\right]^{T}$, and the nominal line flows are (from (5), with $x=0$ ) $y=\left[\begin{array}{lllll}0.27 & 0.73 & 1.09 & 1.18 & 0.09\end{array}\right]^{T}$.

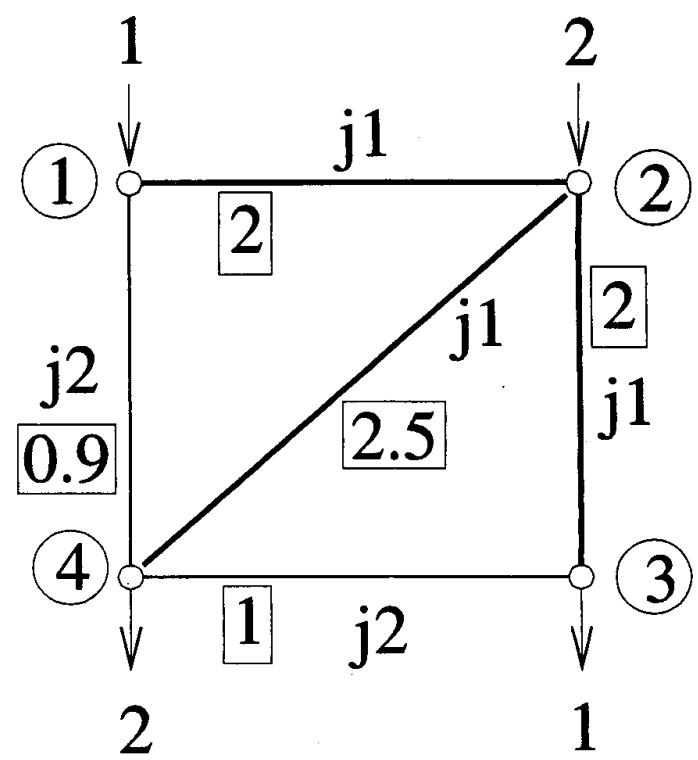

Figure 1: The four bus power system under consideration.

In the case with no loss constraint (i.e., with (9) subject to (10)-(11)) the constraints are linear in $x$. The solution is $x=-0.4$, for either criterion function $I(x)$. This results stresses the importance of a high-capacity network, as the generator \#2 is much better connected with loads \#3 and \#4.

In DC models of power systems it is assumed that branch resistances are small compared with reactances (typically $\leq 10 \%$ ), so they are disregarded in loop flow calculations. The resistances are, however, needed for real power loss calculations, so we set $R=$ $\operatorname{diag}\left(\left[\begin{array}{lllll}0.10 & 0.20 & 0.10 & 0.05 & 0.20\end{array}\right]\right)$. With $x=-0.4$, the losses equal $285.5 e^{-3}$. If we pose the optimization problem (9) with all three constraints (10)-(12)) and with $W=280 e^{-3}$, then the rescheduling of the first generator $x$ has to increase in magnitude to $x=-0.48$.

\section{A BENCHMARK EXAMPLE}

For our benchmark example, we use the transmission network of the standard IEEE 14 bus test case. In this system $G=5, D=9$, and $\breve{L}=20$. We consider outage of all lines, except the ones corresponding to the three winding transformer in this power system (since this outage would correspond to a loss of system connectedness, or integrity), so $K=15$. We generalize the original test case by replacing synchronous condensers with generators, and we allow non-zero loads in all remaining buses. We display the network diagram in Fig. 2.

In this benchmark example $\breve{p}_{g}=\left[\begin{array}{lllll}2 & 1.5 & 1 & 1.25 & 0.75\end{array}\right]$, we assume that line flows are limited (in magnitude) to $\bar{y}=1.91$; there is a total of 328 constraints in the problem, and unknown $x$ is a 4 -dimensional vector. The 


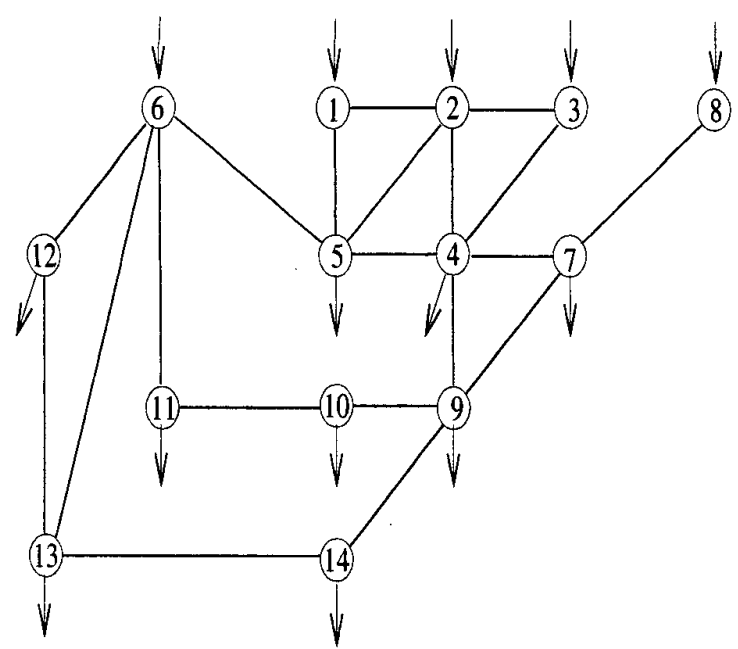

Figure 2: The network diagram of the IEEE 14 bus benchmark.

solution turns out to be

$$
x=\left[\begin{array}{llll}
-0.100 & 0.025 & 0.025 & 0.025
\end{array}\right]^{\top}
$$

We can interpret this solution in terms of the network topology and flow constraints - in the case of outage of either of the lines connected to bus \#1 (where the first generator is located), and given the injection of 2 units, the remaining line will be overloaded (by $2-1.9=0.1$ ). That is exactly the reduction in generation in bus \#1 requested by the first component of $x$; since there are no other active constraints, the net change is apportioned equally among the remaining generators.

In applications to large scale power systems some parts are likely to be aggregated into zones (or super nodes) within which there are no significant flow constraints. While this would decrease the size of the problem, good scaling properties of the proposed algorithm are critical for its maturation into an industry-grade methodology.

\section{CONCLUSIONS}

The paper addresses the issue of improving transmission security in a deregulated power market. We propose an optimization procedure that assures that transmission security is maintained, and generates minimal corrections in existing contractual transactions that are necessary to ensure security. The main computational tools belong to the family of convex optimization methods, and are characterized by a non-explosive growth when the problem size increases. As such, they have a potential of being valuable instruments in the armamentarium of energy engineers, while broadening the application domain of tools developed in the control domain.

\section{ACKNOWLEDGMENTS}

AMS is indebted to the National Science Foundation for support under Grants ECS-9502636 and ECS-9820977 (AMS); AMS also thanks the Office of Naval Research for support under Grant N14-97-1-0704.

\section{REFERENCES}

[1] Ilic, M., F. Galiana, and L. Fink, Eds., Power System Restructing, Engineering and Economics, Kluwer Academic Publishers, Boston, 1998.

(2) North American Reliability Council, Available transfer capability definitions and determiniation, Technical report, NERC, June 1996.

[3] Gross, G., Ed., Proceedings of the workshop on available transfer capability, Urbana, IL, June 1997.

[4] Fang, R.A., A.K. David, "Transmission Congestion Management in an Electricity Market," IEEE Transactions on Power Systems, Vol. 14, No. 3, August 1999, pp. 877-883.

[5] S. Boyd, L. El Ghaoui, E. Feron, V. Balakrishnan, Linear Matrix Inequalities in System and Control Theory, SIAM, 1994. 